\title{
Atypical placement of totally implantable venous access device in paediatric patients - case reports
}

\author{
Aleksandra Kotowska, Zuzanna Lewandowska, Patrycja Sosnowska, Sebastian Moryciński, \\ Katarzyna Kmieciak, Przemysław Mańkowski \\ Department of Paediatric Surgery, Traumatology, and Urology, Poznan University of Medical Sciences, Poznan, Poland
}

\section{ABSTRACT}

Central line catheterisation plays a crucial role in prolonged treatment in children. Totally implantable venous access devices (TIVADs) are associated with a lower risk of thrombosis and are perceived by patients as more comfortable to use. The catheterisation is most commonly performed through the internal jugular vein or the subclavian vein.

The present article shows the cases of two patients in whom atypical placement of a TIVAD was used. The first patient underwent TIVAD placement via femoral vein due to tumour mass in the thoracic cavity and thrombotic stenosis of both jugular and subcostal veins. In the second case, atypical localisation applied to the placement of a reservoir in the left parasternal region. In both patients, the atypical placement of TIVAD resulted in prolonged patency and provided access for further treatment.

\section{KEY WORDS:}

TIVAD, central catheter, children.

\section{INTRODUCTION}

Central line catheterisation is a widely used procedure in many fields of paediatrics. Amongst them, long-term or prolonged appliance of intravenous medication (e.g. chemotherapy) is one of the most important reasons for implantation $[1,2]$.

In comparison to a peripherally inserted central catheter (PICC lines), totally implantable venous access devices (TIVADs), commonly known as ports, cause fewer complications after insertion. For instance, the risk of thrombosis is significantly lower in patients with a port than in those with a PICC line [3]. It allows long-term treatment. Self-evaluated satisfaction of port usage is very high, with up to $92 \%$ of patients being satisfied with TIVAD and eager to recommend this way of taking medication to other patients in need [4].
The most popular routes of insertion are the internal jugular vein and the subclavian vein. The choice depends on the preferences of the surgeon performing the procedure, common practices in a particular hospital, or indications and contraindications to a certain approach [5]. Femoral vein cannulation is believed to cause more infectious complications, but the possibility of causing iatrogenic pneumothorax is avoided [6].

\section{CASE REPORTS}

A seven-year-old boy was admitted to the Department of Paediatric Oncology, Haematology, and Transplantology due to chronic rhinorrhoea, cough, and numerous dilated vessels on the surface of the thorax.

According to his medical record, the patient was diagnosed with acute lymphoblastic leukaemia (ALL) four

\section{ADDRESS FOR CORRESPONDENCE:}

Przemysław Mańkowski, Department of Paediatric Surgery, Traumatology, and Urology, Poznan University of Medical Sciences, 27/33 Szpitalna St., 60-572 Poznan, Poland, e-mail: mankowskip@wp.pl 
years earlier and had been treated in accordance with the ALL IC BFM 2002 protocol since then. ALL remission was achieved after a year of treatment.

During hospitalisation, the physical examination revealed facial and cervical oedema. The numerous café au lait spots were noticed on the skin of the abdomen. The laboratory tests unveiled anaemia and iron insufficiency. Erythrocyte sedimentation rate and lactic acid level were elevated.

Bone marrow aspiration did not reveal any neoplastic infiltration; however, a large tumour $(42 \mathrm{~mm} \times 50 \mathrm{~mm}$ $\times 61 \mathrm{~mm}$ : ap $\times \mathrm{dc} \times \mathrm{cc}$ ) was localised in the medial and anterior mediastinum in computed tomography. The patient was transferred to Department of Paediatric Surgery, where a biopsy was performed during microthoracotomy a few days later. Pre-T-cell lymphoblastic lymphoma was diagnosed. The chemotherapy was first implemented in EURO-LB 2002 Protocol I. The patient was qualified to TIVAD insertion to continue the chemotherapy. Due to a massive tumour localised in the thoracic cavity and thrombotic stenosis of both jugular veins and subcostal veins, the TIVAD was placed in the right femoral vein.

The procedure was performed under general anaesthesia. The vein was identified by ultrasound guidance and punctured. Then, a catheter was inserted under X-ray guidance. At the end of the procedure, the catheter was combined with Baby-port reservoir, placed subcutaneously on the right inguinal fascia. A radiograph taken after the surgery confirmed the localisation of the catheter's tip in the inferior vena cava.

The patient started chemotherapy in M1-MTX protocol. In the next few months, Euro-LB-02 Protocol II chemotherapy was continued. During the treatment, TIVAD was flushed with heparin regularly once a month. Signs of infection in the area of insertion or catheter malfunction were not observed. In a four-year follow-up, the patient maintained the patency of the port.

Another patient was a boy with diagnosed type A haemophilia. TIVAD was implemented in the right subclavian vein when the patient was three years old. The device was required to continue the supplementation of factor VIII. The first exchange took place when the patient was seven years old, in 2012. The left subclavian vein was first punctured and identified with the use of radioscopy. The tip of the catheter was localised in the superior caval vein, above the right atrium. Then, after catheter insertion, a reservoir was attached to the muscular fascia. The old catheter was removed from the right subclavian vein.

In 2016, the catheter was replaced again due to its malfunction caused by an excessive amount of adipose tissue localised directly over the reservoir.

First, the old TIVAD was removed via a cut in the old postoperative cicatrix. When the catheter and the reservoir were successfully disposed of, the operator punctured and identified left subclavian vein. With the aid of radioscopy, a new catheter was introduced to the superior caval vein. Subsequently, the catheter was combined with a BardPort reservoir, which was placed in the left parasternal region because of thinner subcutaneous tissue and better access for the Huber needle. All of the procedures were performed without any complications.

\section{DISCUSSION}

In paediatric haematological diseases, including both neoplasms deriving from the lymphoreticular system and non-neoplastic issues, providing central venous access is an inevitable procedure in the process of the full-spectrum treatment of a child. Treatment via a central venous catheter, both PICC line and port, can be started at any age. It is used even in preterm infants [7].

In oncology, TIVAD is a preferable option both for young children fully dependant on parents' care and for older ones. It allows minimisation of the amount of time spent taking care of the device. From a psychological point of view, it is very important that it does not restrict physical activity and is not conspicuous. Last but not least, it is connected to a lower number of infectious complications in children during chemotherapy [8].

Ports are favourable to PICC lines also in the context of the frequency of their removal [9]. The advantages of the TIVADs are connected with the smaller amount of physical and psychological discomfort in children during prolonged treatment, and they reduce the possibility of complications interfering with the treatment [10].

The big advantage of TIVAD is that it does not need to be removed and inserted again frequently. For a PICC line, depending on the type of catheter and the reason for placement, the maximum amount of time for removal is between 48 hours (for antibiotic therapy) and up to one year of therapy [11]. For a port, there is no specified time for keeping it inserted, and the only limitation to keeping it persistently are complications such as infection (which can lead to sepsis and is an indication for immediate removal of the port) and thrombosis.

There are several contraindications that should be considered before inserting in the central venous system. Those include: systemic sepsis (with an exception of a necessity to proceed IV antibiotic treatment), local cellulitis, neutro- or thrombocytopaenia or deranged coagulation, state after removing the catheter due to infection or thrombosis, deep venous thrombosis in medical record, post-radiotherapy state of the location of approach, anatomical obstacles $[12,13]$. Taking this into consideration, finding the optimal, safe venous approach in children remains a challenge for clinicians.

As the first case shows, mediastinal lymphomas, the source of $15 \%$ of primary mediastinal masses and $45 \%$ of anterior mediastinal tumours in children, can have a strong influence on the anatomical topography of the thoracic cavity and neck, making it impossible to advance 
the catheter into the jugular vein or the subclavian vein. What is more, recurring haematological neoplasm or coincidental occurrence of two malignancies deriving from the lymphoreticular system, as in this case, can cause problems due to the recurring need of catheterisation. The previously acceptable approach (e.g. brachial vein, which is a popular approach in neonates) can be unacceptable in bigger children or be afflicted by thrombosis. This leads to the requirement of an alternative approach, such as the femoral vein.

The most recommended puncture sites for implantable venous access are the internal jugular vein and the subclavian vein. The subclavian vein seems to be preferred, mostly due to its relatively large lumen, easy access, and acceptable cosmetic effect. Besides, a reservoir secured on the major pectoral muscle does not restrict the mobility of the patient's head and arm, as opposed to a port in the jugular vein [14]. However, this location has many disadvantages, the most common of which are an arterial puncture, catheter-related thrombosis, stenosis of the punctured vessel, and pneumothorax.

There are several clinical situations in which seeking for an alternative place of cannulation is inevitable. Femoral access may be a good choice in patients with superior vena cava syndrome, congenital abnormalities of the superior vena cava, or skin lesions on the neck and chest. Due to anatomical considerations, in the process of cannulation, a femoral artery may be mistakenly punctured. However, it may be avoided by using ultrasound guidance. The reservoir can be placed successfully on the inguinal fascia. During the treatment of the first patient, neither signs of local cellulitis nor complaints about any difficulties of limb motion were noted. Despite being a promising chance to provide a long-lasting intravenous therapy, the femoral approach is suspected of higher risk of infectious and thrombotic complications.

The thrombosis associated with the previous insertion of a PICC line or TIVAD is one of the biggest problems in the continuous treatment of severely ill children.

According to The American Society of Anaesthesiologists Task Force on Central Venous Access, the internal jugular approach for PICC line should be favoured, because of increased risk of thrombosis in both the subclavian and femoral veins. On the other hand, this complication can be diagnosed earlier in the femoral approach due to more visible symptoms of deep vein thrombosis in lower extremity [15].

A retrospective cohort study from North Carolina Children's Hospital revealed that the general frequency of thrombosis cases (both clinically and radiologically found) in children with PICC line and port is comparable, with a slightly higher percentage in the PICC group $(3.6 \%)$ than in the port group $(3.3 \%)$. What is interesting, the same research showed that the femoral and the saphenous vein approaches (grouped together) have the lowest ratio of thrombosis occurrence $(2 \%)$. The highest ratio was represented in the group with cannulated brachial or cephalic vein (3.5\%) [16].

Another research shows, that in the group of patients with TIVAD inserted from femoral access, the thrombotic complications were presented only in $0.23 / 1000$ patients $(0.5 \%)[6]$.

The second major problem that needs to be taken into consideration is the infection risk. It was long believed that the femoral approach is more vulnerable to infectious complications due to its localisation close to the perineal area. However, recent data show that the risk is actually lower, with only $18.6 \%$ of patients showing positive culture tests, while subclavian and jugular veins showed $24.5 \%$ and $28 \%$, respectively [17].

Venous access is important for both non-inhibitorand inhibitor-taking patients with type A haemophilia. Prophylactic infusion of factor VII has become a standard treatment. The therapy requires a reliable approach that can be used repeatedly for a long period of time. In the second case the patient started the supplementation of factor VII since the age of three years. To provide the treatment, TIVAD was implemented first in the right subclavian vein. A study showed that in patients with haemophilia TIVAD enables regular prophylactic or on-demand home treatment with an acceptable frequency of side effects. Usage of a port in children with inhibitor therapy is more frequent; therefore, venous access should be less vulnerable to both thrombosis and infection of the approach site [17].

Nevertheless, it should be considered that the thrombosis risk can vary in conjunction with comorbidities. It is a rare finding in patients with haemophilia $\mathrm{A}$ [18]. The frequency of thrombotic complications was long thought to be very low, up to $2 \%$ [19]. However, radiological examination showed that lots of the patients (up to 87\%) have subclinical thrombosis, which should be observed in a thorough follow-up [20].

Sometimes, especially in the paediatric population, catheter malfunction may be caused by changing anatomic conditions. In the second case, the position of the reservoir needed to be reconsidered due to the accretion of adipose tissue on the chest. Some authors indicate that the gain of weight and height can result in an extravascular relocation of the catheter tip. It is also suggested that the port be implanted in the left subclavian vein, which allows a longer part of the catheter to be placed intravenously [14]. As in the first case report, in older children, in whom the growth does not influence the anatomy strongly, the port can stay inserted for many years, and with proper care (e.g. heparin infusions and flushing with saline) it can persist unobstructed.

\section{CONCLUSIONS}

The general acceptance of TIVAD supports the long maintenance of this access. The patient generally views 
having a TIVAD positively, with $92 \%$ of patients being satisfied with this way of treatment. $74 \%$ of the patients (based on oncologically treated adult patients) are also willing to keep the port after the end of treatment [4].

\section{DISCLOSURE}

The authors declare no conflict of interest.

\section{REFERENCES}

1. Bradford NK, Edwards RM, Chan RJ. Heparin versus $0.9 \%$ sodium chloride intermittent flushing for the prevention of occlusion in long-term central venous catheters in infants and children: A systematic review. Int J Nurs Stud 2016; 59: 51-59.

2. Derenski K, Catlin J, Allen L. Parenteral Nutrition Basics for the Clinician Caring for the Adult Patient. Nutr Clin Pract 2016; 31: 578-595.

3. Patel GS, Jain K, Kumar R, et al. Comparison of peripherally inserted central venous catheters (PICC) versus subcutaneously implanted port-chamber catheters by complication and cost for patients receiving chemotherapy for non-haematological malignancies. Support Care Cancer 2014; 22: 121-128.

4. Ignatov A, Hoffman O, Smith B, et al. An 11-year retrospective study of totally implanted central venous access ports: Complications and patient satisfaction. Eur J Surg Oncol 2009; 35: 241-246.

5. Harish F, Madhu YC. Femoral Port Placement - Report of Two Cases. Indian J Surg Oncol 2011; 22: 31-33.

6. Goltz JP, Janssen H, Petritsch B, Kickuth R. Femoral placement of totally implantable venous power ports as an alternative implantation site for patients with central vein occlusions. Support Care Cancer 2014; 22: 383-387.

7. Caruselli M, Carboni L, Franco F, et al. Central venous catheters in neonates: from simple monolumen to port catheter. J Vasc Access 2011; 12: 4-8.

8. Sola JE, Stone MM, Colombani PM. Totally implantable vascular access devices in 131 pediatric oncology patients. Pediatr Surg Int 1996; 11: 156-158.

9. Mirro J, Rao BN, Kumar M, et al. A Comparison of Placement Techniques and Complications of Externalized Catheters and Implantable Port Use in Children With Cancer. J Pediatr Surg 1990; 25: 120-124.

10. Al-Hathal M, Malmfors G, Garwicz S, Bekassy AN. Port-A-Cath in Children during long term chemotherapy: Complications and Outcome. Pediatr Hematol Oncol 1989; 6: 17-22.

11. Drewett SR. Central venous catheter removal: procedures and rationale. Br J Nurs 2000; 9: 2304-2315.

12. Puppala S, Siegal JA, Windle ML, Scudder L. Central Venous Access via Tunneled Cathether. http://emedicine.medscape.com/article/1375734-overview (access: 17.10.2016).

13. Povoski SP. Long-Term Central Venous Access. http://www.cancernetwork.com/cancer-management/long-term-central-venous-access (access: 17.10.2016).

14. Bucki B, Tomaszewska R, Karpe J, et al. Self- removing of central venous catheter after long time in pediatric oncology patients Onkol Pol 2005; 1: 3-34.

15. Duesing LA, Fawley JA, Wagner AJ. Central Venous Access in the Pediatric Population With Emphasis on Complications and Prevention Strategies. Nutr Clin Pract 2016; 31: 490-501.

16. Smitherman AB, Alexander T, Connelly M, et al. The Incidence of Catheter-Associated Venous Thrombosis in Noncritically Ill Children. Hosp Pediatr 2015; 5: 59-66.
17. van den Berg HM, Fischer K, Roosendaal G, Mauser-Bunschoten EP. The use of the Port-A-Cath in children with haemophilia - a review. Haemophilia 1998: 4: 418-420.

18. Bicer M, Yanar M, Tuydes O. Spontaneous deep vein thrombosis in hemophilia A: a case report. Cases J 2009; 2: 6390.

19. Karapinar B, Cura A. Complications of central venous catheterization in critically ill children. Pediatr Int 2007; 49: 593-599.

20. Izzi G, Franchini M, Bonetti L, Tagliaferri A. The use of central venous catheters in haemophilia patients. Haemophilia 2016; 16 (Suppl 1): 29-31. 